\title{
Diagnóstico de la logística del carbón en Norte de Santander
}

\section{Diagnosis of coal in logistics Norte de Santander}

\section{Diagnóstico da logística do carvão em Norte de Santander}

\author{
Jorge Enrique Rodríguez-Guevara ${ }^{1}$, Adriana Mora-Urbina ${ }^{2}$
}

Forma de citar: J. E. Rodríguez, A. Mora, "Diagnóstico de la logística del carbón en Norte de Santander", Respuestas, vol. 20, no. 1, pp. 30-37, 2015.

Recibido:

4 de Junio de 2014

Aceptado:

3 de Noviembre 2014

\section{Resumen}

Antecedentes: La participación del carbón colombiano en el mercado internacional ha dependido y dependerá fundamentalmente de competir con precios con otros posibles proveedores internacionales, si no se cambia la estrategia a competir. Por ello uno de los factores más importantes a tener en cuenta tiene que ver con los costos de la operación logística, para el caso de Norte de Santander. Objetivo: El objetivo principal de este proyecto es realizar el diagnóstico de la operación logística del carbón en Norte de Santander. Metodología: Básicamente la metodología utilizada fue la aplicación de encuestas a los principales expertos y empresarios exportadores de Carbón en Norte de Santander. Resultados: Uno de los aspectos más relevantes que mitigan la posibilidad de capturar valor en el comercio exterior, son sus altos costos en la operación logística. El problema identificado se da en la ausencia de estudios que permitan caracterizar la distribución física internacional del sector del carbón en Norte de Santander, en los Municipios más representativos para su extracción. Conclusiones: Finalmente el proceso de internacionalización de la economía obliga a los sectores productivos, a pasar de las ventajas comparativas, a ventajas competitivas. Estas últimas son necesarias para aprovechar los mercados internacionales, sin embargo, sectores estratégicos por su potencial en reservas y características de su producto, como el sector del carbón en Norte de Santander, aún no consolidan elementos para su aprovechamiento.

Palabras Clave: Carbón, Diagnóstico, Estrategia, Investigación, Logística.

\begin{abstract}
Background: The participation of Colombian coal in the international market has depended and will depend primarily on price to compete with other potential international suppliers, if the strategy is not changed to compete. Therefore one of the most important factors to consider has to do with the cost of logistics operation, in the case of Norte de Santander. Objective: The main objective of this project is to make the diagnosis of coal logistics operation in Northern Santander. Methods: Basically the methodology used was the application of polls leading experts and entrepreneurs exporters of coal in Norte de Santander. Results: One of the most important aspects that mitigate the possibility of capturing value in foreign trade are high costs in logistics operation. The identified problem occurs in the absence of studies to characterize the international physical distribution of coal in Norte de Santander, the most representative for extraction Municipalities sector. Conclusions: Finally the internationalization of the economy requires productive sectors, to shift from comparative advantages to competitive advantages. These are needed to tap
\end{abstract}


international markets, however, strategic sectors for its potential reserves and features of your product, as the coal sector in Norte de Santander, not yet consolidated elements for their use.

Keywords: Coal, Diagnosis, Strategy, Research, Logistics.

\begin{abstract}
Resumo
Antecedentes: A participação do carvão colombiano no mercado internacional tem dependido e está dependendo fundamentalmente de competir com preços com outros possíveis provedores internacionais, senão se muda a estratégia a competir. Por isto, um dos fatores mais importantes a ter em conta tem que ver com os custos da operação logística, para o caso de Norte de Santander. Objetivo: O objetivo principal deste projeto foi realizar o diagnóstico da operação logística do carvão em Norte de Santander. Metodologia: Basicamente a metodologia utilizada foi a de aplicação de pesquisas de opinião aos principais expertos e empresários exportadores de carvão em Norte de Santander. Resultados: Um dos aspetos mais relevantes que mitigam a possibilidade de capturar valor no comercio exterior, são seus altos custos na operação logística. O problema identificado se dá na ausência de estudos que permitam caracterizar a distribuição física internacional do setor do carvão em Norte de Santander, nos Municípios mais representativos para sua extração. Conclusões: Finalmente, o processo de internacionalização da economia obriga aos setores produtivos a passar das vantagens comparativas às vantagens competitivas. Estas últimas são necessárias para aproveitar os mercados internacionais, no entanto, setores estratégicos por seu potencial em reservas e características de seu produto, como o setor do carvão em Norte de Santander, ainda não consolidam elementos para seu aproveitamento.
\end{abstract}

Palavras-Chave: Carvão, Diagnóstico, Estratégia, Logística, Pesquisa.

\section{Introducción}

El proceso de inserción de la economía nacional en los mercados internacionales, y cada tendencia de integración del país con economías más dinámicas, a su vez, la disminución en la distancia económica entre los países, plantea un escenario global cada vez con mayor interdependendencia. Esto se ve reflejado en los sectores donde se cuentan con ventajas comparativas, que van perdiendo fuerza en el posicionamiento de los sectores que antes, eran líderes por su diferenciación comparativa. Uno de ellos, es el sector del carbón, que es considerado uno de los sectores a ser protagonista en el desarrollo regional en Norte de Santander. A pesar de lo anterior, en los últimos años se propuso la puesta en marcha el fortalecimiento del carbón para Norte de Santander, Sin embargo este aún no ha podido consolidar. [1]
Referentes como la mejora continua aplicado a la logística, será la bitácora que permitirá guiar el estudio sobre las posibilidades de aportar al desarrollo local, con aspiraciones globales.

Por esto, el objetivo del estudio propuesto es realizar el diagnóstico de la operación logística y de distribución física internacional del carbón en Norte de Santander. Para ello, se plantean tres objetivos específicos que son: el primero, identificar los eslabones que componen el proceso logístico del sector del carbón en su funcionamiento en el Departamento; el segundo, describir los elementos que caracteriza la distribución física internacional del sector del carbón en Norte de Santander; y el tercero, proponer con base a los resultados obtenidos en el diagnóstico las mejoras pertinentes en las operaciones de logística y distribución 
No. 1

Enero - Junio 2015 ISSN 0122-820X

PP: $30-37$ internacional del carbón para fortalecer las ventajas competitivas.

Lo anterior, se logrará indagando fuentes claves para el estudio, a su vez, cotejando la información suministrada, con expertos sobre el sector y las operaciones logísticas del mismo. Los resultados esperados, están enmarcados con la caracterización de la distribución física internacional llevada a cabo en los municipios en mención, para proponer las mejoras pertinentes en la operación logística más adecuada, y de esta forma, aportar al desarrollo de ventajas competitivas para la región [2].

Considerando que en el departamento el desarrollo industrial es precario en comparación con otras regiones del país, y que el costo económico y de tiempo que se requiere para su eventual progreso es alto, el desarrollo del sector del carbón surge como una oportunidad interesante porque es una de las actividades más dinámicas de la economía regional, ya que es uno de los grandes empleadores, en empleos directos como indirectos; es un generador importante de divisas y de niveles de inversión, en lo extranjera como nacional; un gran aportante a los ingresos regionales vía regalías, lo que lo ubica como un sector que tiene mucho que aportar al desarrollo local [3],[4].

Además, los actuales sistemas de distribución física, aún se basan en procesos que no permiten alcanzar estándares de eficiencia, afectando el precio final del producto, de manera sensible hacia los consumidores [5], [6].

Sin embargo, para que la implementación sea exitosa se requiere apoyar los esfuerzos de los actores mediante la asesoría y capacitación desde la academia, con estudios que fortalezcan la creación de captura de valor en los encadenamientos productivos y su correspondiente logística, logística que cada vez más se ve afectada por la infraestructura vial paupérrima del Departamento, y que no corresponde con el camino adecuado en la eficiencia del sector [7], [8], [9].

En la actualidad, los mercados internacionales no han sido favorables para el sector, presentando una tendencia a la baja del precio internacional del carbón, consecuencia de la desaceleración de las economías como la de Estados Unidos, Europa, y los mismo países asiáticos, principalmente China, que en los últimos años crece, pero no con la misma tendencia de principios de la primera década del S XXI [10].

\section{Materiales y métodos}

\subsection{Materiales}

La orientación metodológica se trata de una propuesta donde el uso de enfoque se da desde una perspectiva de población o muestra seleccionada: el grupo población que se trabajo es el conjunto de empresas que hacen parte de la cadena productiva del carbón en Norte de Santander, que se encuentran legalmente constituidas en la Cámara de Comercio de Cúcuta, como extractores, comercializadores, productores, distribuidores de carbón, en los municipios en mención (El Zulia, Sardinata, Cúcuta, Tibú) [11].

\subsection{Métodos}

La metodología que se utilizó contempla las encuestas, revisiones bibliográficas sobre algunos temas que se relacionan con el proyecto planteado.

El lugar donde se desarrolló gran parte de la investigación, son las instalaciones de la Universidad de Santander (UDES) Sede Cúcuta. Programa Comercio Exterior. Semillero de Investigación SEMICOMEX y Grupo de Investigación FENIX UDES. 
Así como los lugares representados por extractores, productores, comercializadores, Distribuidores del Departamento Norte de Santander ubicados en los municipios de Cúcuta, Sardinata, Tibú y Zulia. De la misma manera serán visitados gremios, asociaciones y demás instituciones que hacen parte de la cadena productiva del carbón en el Departamento Norte de Santander.

\subsubsection{Método A.}

En el presente estudio se utilizó la encuesta, mediante un cuestionario, el cual se aplicará a todos los eslabones de la cadena de distribución física internacional, con preguntas mixtas (abiertas y cerradas). Se utilizó la escala de Likert para su valoración:

1. Totalmente en desacuerdo

2. En desacuerdo

3. Ni de acuerdo ni en desacuerdo

4. De acuerdo

5. Totalmente de acuerdo.

Este ordenamiento racional se originó a partir del cuadro de operacionalización de variables. Un porcentaje considerable de opciones, tuvo la posibilidad de responder otra, cual, lo que garantiza que no se presente un sesgo considerable con las opciones presentadas en el instrumento de recolección.

El muestreo que se trabajó se calculó según el total de la población de empresas involucradas en la cadena productiva legalmente constituida en la Cámara de Comercio de Cúcuta, así:

$$
n=\frac{N * Z^{2} * p * q}{d^{2} *(N-1)+Z^{2} * p * q}
$$

Donde

$n=\frac{70 *\left((1,645)^{2} * 0,15 * 0,90\right.}{0.20^{2} *(70-1)+0,90^{2} * 0,15 * 0,90}$

$n=$ Tamaño de la muestra, 70 empresas

$z=$ Nivel de confianza. 1.645 al cuadrado (si la seguridad es del 90\%)

$p=$ Frecuencia esperada del factor a estudiar. $($ En este caso $1-0.10=0.90)$

$q=1-p$. proporción esperada (en este caso $15 \%=0.15$ )

$d=$ precisión (en su investigación use un 20\%).

$$
n=8
$$

Para esta investigación se utilizaron una muestra de 8 empresas.

\subsubsection{Método B.}

Otro de los instrumentos que se aplicó, se encuentra la lista de cotejo o chequeo, para verificar lo ideal con la situación real que se presentó. (si-no).

También se procedió a realizar una validación de contenido por juicio de expertos, para ello se le envió el instrumento a un metodólogo y a un especialistas en el área logística internacional.

\section{Resultados y análisis}

Se muestra claramente los resultados obtenidos de las encuestas y entrevistas aplicadas a las 8 empresas productoras de carbón con el propósito de arrojar la información que ayude a identificar el diagnostico actual de la logística empresarial en Norte de Santander

\subsection{Análisis de encuestas}

En esta parte se desarrolló el primer objetivo que se refiere al análisis de la operación 
No. 1

Enero - Junio 2015 ISSN 0122-820X

PP: $30-37$ logística del carbón en Norte de Santander; debido a que estas encuestas se aplicaron a expertos logísticos de la región.

Al tabular la información de las encuestas realizadas se puede dar como resultados lo siguiente:

El principal canal de distribución que utiliza la empresa es de tipo directo Extractorconsumidor, debido a que lo que la mayoría de las empresas hacen es sacar el carbón y exportarlo.

Así mismo las empresas prefieren invertir en un volumen elevado de sus recursos tanto en infraestructura como en tecnología de punta, porque estas inversiones ayudarían a que su empresa tuviera un desarrollo más rápido y competitivo, debido a las altas exigencia del mercado internacional.

Porotro parte laprincipal estrategiaque utilizan las empresas para ser más competitivos es la calidad del producto debido a que el carbón de nuestro departamento posee características físico-químicas que permiten utilizarlo para diversas actividades como en las termoeléctricas para la generación de energía, lo que ayuda a suplir de cierta forma las altas exigencia del mercado internacional, también se destaca la tecnología y la innovación en los procesos, como estrategia.

Otro de los resultados que arrojo las encuestas fue que las empresas no creen que la creación o instalación de una nueva empresa ya sea competencia directa o producción de un bien sustituto afecte directamente su empresa, debido a que esta empresa ayudaría a ampliar el gremio y también a posicionar el carbón en el mercado mundial.

También se destaca el nivel de innovación que tiene cada empresa, la mayoría desarrollo un sistema de innovación medio o bajo, es decir no hay suficiente inversión para este sector, lo que impide que los procesos sean eficazmente.
Por otro lado la mayoría de empresas no cuentan con ninguna certificación de calidad, debido a que aún no es requisito obligatorio para la exportación del producto en Colombia, algunas empresas cuentan con la certificación ISO 9000, versión 2008.

El principal factor que ha afectado notablemente el sector del carbón ha sido los precios internacionales, debido a que los precios han tenido una disminución a nivel internacional.

Otro de los resultados arrojado es que las empresas están actualizándose diariamente de los acontecimientos que ocurren en el mercado actual, ya que este mercado está cambiando continuamente.

Por último los carbones más demandados por el mercado internacional son los coquizables, los carbones térmicos también son representativos en el mercado y la capacidad productiva que tienen la mayoría de las empresas está entre 2000 y 65000 Tn mensualmente, esta información es la resultante de las encuestas aplicadas.

\subsection{D.O.F.A. del carbón en Norte de Santander}

En esta parte se desarrolla uno de los objetivos específicos describir los elementos que caracteriza la distribución física internacional del sector del carbón en Norte de Santander.

Se explica claramente cada una de las debilidades, oportunidades, fortalezas, y amenazas del sector carbón en Norte de Santander. 


\begin{tabular}{|c|c|}
\hline DEBILIDAD & OPORTUNIDAD \\
\hline $\begin{array}{l}\text { - No se cuenta con un adecuado almacenamiento, es decir no hay } \\
\text { - Lepósitos indicados para guardar el carbón mientras se exporta. } \\
\text { cas vías de transporte de acceso a las minas están en malas } \\
\text { condiciones. } \\
\text { La poca participación del Estado en desarrollar una infraestructura } \\
\text { vial. } \\
\text { El incremento de los costos de transporte para extraer el carbón de las } \\
\text { minas. }\end{array}$ & $\begin{array}{l}\text { - La alternativa de la construcción de una nueva ruta internacional: Cúcuta, la fría, } \\
\text { Maracaibo, ya que esto produciría la reducción de costos de transporte. } \\
\text { La terminación de la vía Tibu-La Mata. } \\
\text { El diseño del proyecto de gobierno Nacional del Puerto para facilitar el acceso a } \\
\text { los puertos Colombianos. } \\
\text { Su ubicación geográfica ya que el carbón se puede sacar por el lago de } \\
\text { Maracaibo lo que en otros sectores del país no tienen y no pueden, y así sus } \\
\text { costos de transportes serian relativamente bajos comparados con los precios de } \\
\text { transporte colombiano. } \\
\text { Se puede aprovechar el rio magdalena como una vía que permita costos de } \\
\text { transporte bajos. } \\
\text { Cercanía con el lago de Maracaibo ya que se puede sacar la producción por ahí y } \\
\text { lo que permite la reducción de costos. }\end{array}$ \\
\hline FORTALEZA & AMENAZA \\
\hline $\begin{array}{l}\text { - } \quad \text { Su ubicación geográfica permite ser un departamento fronterizo. } \\
\text { - } \quad \text { Gran oferta de vehículos para transportar el carbón. } \\
\text { El parque automotor. }\end{array}$ & $\begin{array}{l}\text { - Su infraestructura y las vías de comunicación que no permite que sea } \\
\text { competente. } \\
\text { Impacto nacional e internacional en producción y precio lo que causa la } \\
\text { disminución de la demanda. } \\
\text { La inestabilidad del gobierno Venezolano, ya que por ahí es donde se saca la } \\
\text { mayor parte del producto. } \\
\text { - Salidas dependiente con Venezuela, ya que por ahí es el único camino que los } \\
\text { costos salen bajos. } \\
\text { Los costos de transportes colombianos son altos debido a su ubicación referente } \\
\text { a los puertos de Colombia. } \\
\text { - Los puertos se encuentran lejos de los lugares de producción. } \\
\text { En Colombia no hay fletes de compensación. }\end{array}$ \\
\hline
\end{tabular}

La elaboración de la D.O.F.A se realizó con la información recolectada y analizada a partir de las encuestas realizadas a los expertos en Carbón en Norte de Santander.

\subsection{Nuevas necesidades en el sector para nuevas líneas de investigación}

A partir de la investigación se identificaron diferentes necesidades en el sector, las cuales en el resumen siguiente se darán a conocer

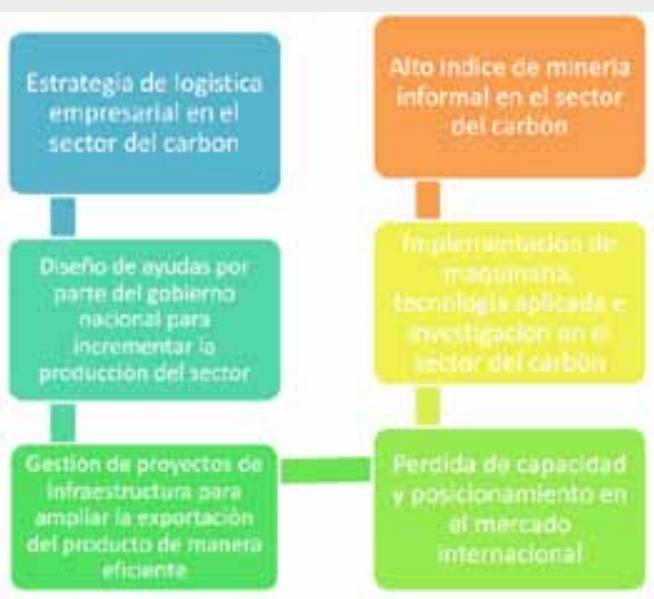

Figura 2. Resumen de las nuevas necesidades del sector carbón. Fuente: Autores

\section{Conclusiones}

Finalmente se concluye que la deteriorada infraestructura de tanto las vías primarias como secundarias afecta en gran medida el transporte y distribución, lo cual no permite obtener gran utilidad al momento de exportar el producto, ya que los costos se incrementan.

Falta de aplicación de modelos de mejoramiento continuo en el sector carbón debido a que estas estrategias permiten mejorar de forma continua y así mismo desarrollar un producto apetecido por el mundo global.

Al finalizar el análisis de datos una grave problemática que es la falta de puertos de fácil acceso, con alta capacidad productiva que permitan la exportación de carbón, y la disminución de los precios de transporte $\mathrm{y}$ distribución del producto, en este caso el carbón.

Por otra parte debido a los costos de transporte y distribución hay posibilidades de exportar por Venezuela pero debido a la inestabilidad de este país, no es posible enviar siempre el producto por estos puertos. 
No. 1

Enero - Junio 2015 ISSN 0122-820X

PP: $30-37$
La Falta de innovación en los procesos de producción del carbón, debido a que se utilizan procesos rudimentarios pero aun así la capacidad es alta para ser uso para hacer uso de estos procesos, lo que conlleva a estar en desventaja con los demás productores de carbón a nivel internacional.

La minería ilegal afecta al gremio carbonero debido a que está en aumento, lo que de una $\mathrm{u}$ otra forma coloca en desventaja el precio debido a que se oferta a menor valor del que se debería vender por las propiedades que posee.

\section{Agradecimientos}

Primero que todo a Dios por permitirnos ejecutar este proyecto, después a la universidad que nos permitió acceder a investigaciones las cuales sirvieron como base para iniciar nuevas propuestas y por último a todos los que nos colaboraron con información necesaria para cumplir los objetivos planeados.

\section{Referencias}

[1] Gobernación del Departamento Norte de Santander. Plan de desarrollo para el Norte de Santander 20122015, un Norte pa'lante. Corredores Logísticos y Zonas Francas Sector Carbón. 2012. [Online]. Disponible en: http://cdgrdnortedesantander.gov.co/ wp-content/uploads/2014/01/plan-dedesarrollo-Norte-de-Santander-20122015-Un-norte-Pa\%C2\%B4lante.pdf.
[5] M.E. Porter. Ventaja Competitiva: Creación y Sostenimiento de un desarrollo superior. Piramide 2010.

[6] M.E. Porter. La ventaja competitiva de las naciones. Argentina: Editorial Vergara, 1991.

[7] República de Colombia, Ministerio de Minas y Energía. Estudio Técnico Sectorial "Infraestructuradel Transporte Multimodal y de Logísticas Integradas para el desarrollo de la Industria Minera en Colombia, con énfasis en Puertos". República de Colombia, Ministerio de Minas y Energía, La Cadena del Carbón. Bogotá 2011. [Online]. Disponible en: http://www.simco.gov.co/LinkClick.as $\mathrm{px}$ ?fileticket $=0 \mathrm{CBLMjONx} 3 \mathrm{M}=\& \mathrm{tab}$ $\mathrm{id}=282$.

[8] J. G. Taboada. El Camino hacia la Competitividad del Carbón. 2010.

[9] C. P. Rodríguez, L. D. Santaella, "Proceso logístico de transporte en la empresa carbones colombianos del Cerrejón S.A" Tesis de Grado Administrador de Negocios Internacionales, Universidad de La Sabana, Chía, 2006. [Online]. Disponible en: http://intellectum.unisabana.edu.co/ bitstream/handle/10818/5949/127750. pdf? sequence $=1$.

[10] L. R. Amortegui, G. Y. Carvajal, "Propuesta de una iniciativa de Cluster. El caso del Cluster del carbón del norte de Cundinamarca, CCNC". Archivos de Economía. República de Colombia, Departamento Nacional de Planeación, Dirección de Estudios Económicos. 2006. [Online]. Disponible en: https:// www.yumpu.com/es/document/ view/14759165/archivos-de-economiadepartamento-nacional-de-planeacion.

[4] M.E. Porter. Ser Competitivo. Barcelona: Deusto S.A Ediciones 2009. 
[11] Dirección de Síntesis y Cuentas

Enero - Junio 2015 Nacionales - DANE 2013 [Online]. Disponible en: https://www.dane.gov. ISSN 0122-820X co/files/investigaciones/boletines/ech/ ml_depto/Boletin_dep_13.pdf. 